\title{
Author Correction: COVID-19 vaccine guidance for patients with cancer participating in oncology clinical trials
}

Aakash Desai, Justin F. Gainor, Aparna Hegde, Alison M. Schram, Giuseppe Curigliano id, Sumanta Pal, Stephen V. Liu, Balazs Halmos, Roman Groisberg Di, Enrique Grande, Tomislav Dragovich, Marc Matrana, Neeraj Agarwal, Sant Chawla, Shumei Kato, Gilberto Morgan, Pashtoon M. Kasi (D), Benjamin Solomon, Herbert H. Loong, Haeseong Park, Toni K. Choueiri(D, Ishwaria M. Subbiah (D), Naveen Pemmaraju, Vivek Subbiah (D) and the COVID 19 and Cancer Clinical Trials Working Group*

Correction to: Nature Reviews Clinical Oncology https://doi.org/10.1038/s41571-021-00487-z, published online 15 March 2021.

In the original version of this Perspective, the name of the author Giuseppe Curigliano was incorrectly written as Guiseppe Curigiliano. The affiliations have been corrected in the HTML and PDF versions of the manuscript.

*A list of authors and their affiliations appears online.

https://doi.org/10.1038/s41571-021-00503-2 I Published online 23 March 2021

(๑) Springer Nature Limited 2021 